\title{
Material Design of Functionally Graded Plates with the Function of Electromagnetic Noise Suppression
}

\author{
(Quantitative Evaluation of the Effect of
} Electromagnetic Noise Suppression)*

\author{
Yoshihiro SUGANO** and Satoshi TAKAHASHI*** \\ **Department of Mechanical Engineering, Iwate University \\ 4-3-5 Ueda, Morioka-shi, Iwate 020-8551, Japan \\ *** Department of Mechanical Engineering, Ishinomaki Senshu University \\ 1 Shinmito, Minamisakai, Ishinomaki-shi, Miyagi 986-8580, Japan \\ E-mail: s-takahashi@isenshu-u.ac.jp
}

\begin{abstract}
The development of materials to suppress electromagnetic noise is in demand. In this paper, we present a method for the material design of functionally graded material (FGM) plates with the function of electromagnetic noise suppression. The FGM plates are considered to be multilayered plates in which the material properties are homogeneous inside each layer. Therefore, the approximate analytical solutions of electromagnetic fields in the FGM plates subject to electromagnetic noise are derived. The expressions for electromagnetic noise suppression are then obtained based on the above analytical solutions. Numerical calculations are carried out for epoxy resin/titanium oxide FGM plates with graded composition distribution expressed in the form of a power function. The effects of graded composition and plate thickness on the electromagnetic noise suppression are quantitatively evaluated, and the material design suitable for the suppression of electromagnetic noise is discussed.
\end{abstract}

Key words: Functionally Graded Material (FGM), Material Design, Analytical Solution, Electromagnetic Noise Suppression

\section{Introduction}

Electromagnetic interference (EMI), which is generated by electromagnetic noise radiated from electronic devices, is of great concern, particularly with the sophistication of the electronic industry. In this paper, we define electromagnetic plane waves that propagate in free space and exert an electromagnetic influence on devices in the form of electromagnetic noise. Despite the abovementioned concerns, many plastics that have little resistance against electromagnetic noise are used as materials for electronic devices that require reliability because of the advantages of the reduction in device size and weight. Measures for the suppression of electromagnetic noise are generally less popular because they are independent of the active functions of the electronic devices. However, such measures are important quality requirements for electronic devices. In the U.S. and Europe, stringent regulations for electromagnetic noise levels have been formulated. Further, reports on the adverse effects on the human body (such as electrical hypersensitivity) with exposure to the electromagnetic waves ${ }^{(1)}$ have been published. Thus, the measures for electromagnetic noise suppression have become the key issue in environmental programs. For 
electromagnetic noise suppression, electromagnetic compatibility (EMC), which includes both the emission abatement of electromagnetic noise and the immunity to electromagnetic noise, is required. In particular, the development of materials to achieve EMC in highfrequency bands is necessary because the development of electronic devices and communication tools that use the microwave and millimeter wave bands has remarkably increased recently ${ }^{(2),(3)}$.

Dielectric loss materials have been used as one of the effective means to achieve $\mathrm{EMC}^{(4)}$. Dielectric loss materials are composite materials that comprise an insulator matrix (such as plastic and ceramic) and resistivity particles (such as metal and carbon black). Electromagnetic noise is suppressed by the process of conversion from electromagnetic energy to thermal energy and the interference of electromagnetic waves caused by the electromagnetic properties of the materials. For increasing the use of electromagnetic waves, materials for electromagnetic noise suppression are applied in a wide variety of environments such as ships, airplanes, and biomedical devices. Hence, it is important to discuss the corrosion resistance, aging effect, and heat and humidity strength of the materials (5), (6). Therefore, the application of functionally graded materials (FGMs) with multiple functions is considered for electromagnetic noise suppression. For multilayer graded plates with material composition expressed as a function of gradual variation, the experiment on the properties of electromagnetic wave absorption for electromagnetic waves reflected from a metal structure was reported by Liu et al. ${ }^{(7)}$. According to their report, the multilayer graded plates showed considerable absorbability for broadband frequencies and the reduction in the plate thickness and weight. However, there so far have been no reports on a theoretical analysis of the FGMs based on Maxwell's equations and a material design for the FGMs with the function of electromagnetic noise suppression.

Therefore, we conduct a theoretical analysis of electromagnetic noise suppression of the FGM plates that have arbitrary nonhomogeneity of electromagnetic properties only through the plate thickness. Numerical calculations are then carried out for the FGM plates composed of epoxy resin matrix and titanium oxide particles by assuming that the FGM plate has a graded composition distribution of the resistivity particles expressed in the form of a power function. Furthermore, the effects of graded composition and plate thickness on electromagnetic noise suppression are quantitatively evaluated.

\section{Electromagnetic Field Analysis of FGM Plate}

\subsection{Analytical model and formulation of electromagnetic field}

In this section, we consider the function of electromagnetic noise suppression of the FGM plate that has arbitrary nonhomogeneity of electromagnetic properties only through the plate thickness ( $z$-axis). The governing equation for the electromagnetic field $\dot{\boldsymbol{E}}$ and $\dot{\boldsymbol{H}}$ in the FGM plate can be expressed on the basis of Maxwell's equations, as follows:

$$
\left.\begin{array}{l}
\nabla \times \dot{\boldsymbol{E}}(\boldsymbol{r}, t)+\dot{\mu}(z) \frac{\partial \dot{\boldsymbol{H}}(\boldsymbol{r}, t)}{\partial t}=0 \\
\nabla \times \dot{\boldsymbol{H}}(\boldsymbol{r}, t)-\dot{\varepsilon}(z) \frac{\partial \dot{\boldsymbol{E}}(\boldsymbol{r}, t)}{\partial t}-\sigma(z) \dot{\boldsymbol{E}}(\boldsymbol{r}, t)=0
\end{array}\right\}
$$

In the above equations, $\boldsymbol{r}$ is the position vector; $\dot{\varepsilon}(z), \dot{\mu}(z)$, and $\sigma(z)$ denote the complex permittivity, complex permeability, and electric conductivity, respectively. The symbols with dots denote the complex quantities. Equation (1) is a simultaneous differential equation with variable coefficients. Hence, deriving the exact solutions for Eq. (1) is very difficult. For electromagnetic field in the FGM plate that has arbitrary nonhomogeneity, exact solutions cannot be obtained. In this paper, the FGM plate is considered to be a 
multilayered plate in which the electromagnetic properties are homogeneous inside each layer, as shown in Fig. 1. The reflected and transmitted waves on the FGM plate are defined as the electromagnetic noise, as stated in the introduction. Thus, the analytical solution for the electromagnetic noise suppression effect of the FGM plate, which is based on the reflection coefficient of the electromagnetic noise at $z=z_{0}$ and the transmission coefficient of the electromagnetic noise at $z=z_{n}$, is derived. It is assumed that the FGM plate is an infinite plate that is divided into $n$ layers having distinct homogeneous properties in each layer, and the FGM plate is irradiated by electromagnetic noise with angular frequency $\omega$ and incident angle $\theta_{0}^{\mathrm{I}}$ from the medium 0 . Superscripts I, R, F, B, T in this analytical model denote the incident, reflected, forward, backward, and transmission waves, respectively. In this analysis, we treat the incident electromagnetic noise as an electromagnetic plane wave in the TE mode (the TE mode does not have electric field in the direction of propagation.), and it is defined as

$$
\left.\begin{array}{l}
\dot{\boldsymbol{E}}_{i}(\boldsymbol{r}, t)=\dot{\boldsymbol{E}}_{i}(\boldsymbol{r}) e^{j \omega t} \\
\dot{\boldsymbol{H}}_{i}(\boldsymbol{r}, t)=\dot{\boldsymbol{H}}_{i}(\boldsymbol{r}) e^{j \omega t}
\end{array}\right\}
$$

Therefore, the electromagnetic field in each medium and each layer satisfies the following governing equation and boundary condition:

$$
\left.\begin{array}{l}
\nabla \times \dot{\boldsymbol{E}}_{i}(\boldsymbol{r})+j \omega \dot{\mu}_{i} \dot{\boldsymbol{H}}_{i}(\boldsymbol{r})=0, \quad(i=0 \sim n+1) \\
\nabla \times \dot{\boldsymbol{H}}_{i}(\boldsymbol{r})-\left(j \omega \dot{\varepsilon}_{i}+\sigma_{i}\right) \dot{\boldsymbol{E}}_{i}(\boldsymbol{r})=0, \quad(i=0 \sim n+1)
\end{array}\right\}
$$

In the above equations, $j$ is the imaginary unit, and subscript $i$ denotes the $i$-th medium or layer. To solve Eqs. (3) and (4), the condition that the electromagnetic wave in the medium $n+1$ is propagated only in the $+z$ direction is included. Further, the incident electromagnetic noise in the TM mode (the TM mode does not have magnetic field in the direction of propagation.) can be analyzed in a similar manner.

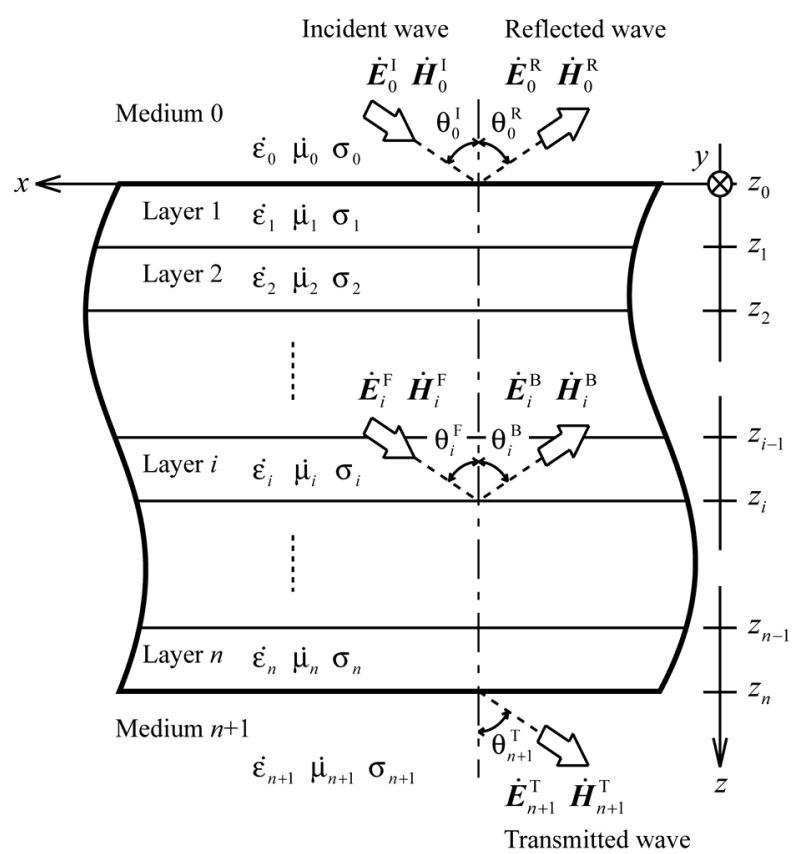

Fig. 1 Analytical model of the FGM plate subject to electromagnetic noise 


\subsection{Analytical solution for electromagnetic field}

We derive the electromagnetic field in each medium and layer. For the electronic field, the following Helmholtz-type differential equation is obtained from Eq. (3):

$$
\nabla^{2} \dot{\boldsymbol{E}}_{i}(\boldsymbol{r})+\left(\omega^{2} \dot{\varepsilon}_{i} \dot{\mu}_{i}-j \omega \dot{\mu}_{i} \sigma_{i}\right) \dot{\boldsymbol{E}}_{i}(\boldsymbol{r})=0
$$

By expressing Eq. (5) with the Cartesian coordinate system, as shown in Fig. 1, the general solution for the electric field can be obtained by the separation of variables method. Moreover, by substituting the solution of electric field into Eq. (3) and differentiating the equation, the general solution for the magnetic field can be obtained. Therefore, the required electromagnetic fields in the FGM plate are expressed as

$$
\begin{aligned}
\dot{E}_{y i} & =\dot{E}_{y i}^{\mathrm{F}}+\dot{E}_{y i}^{\mathrm{B}}=\left\{\dot{A}_{i} e^{-\dot{\gamma}_{i}\left(-x \sin \theta_{i}^{\mathrm{F}}+z \cos \theta_{i}^{\mathrm{F}}\right)}+\dot{B}_{i} e^{-\dot{\gamma}_{i}\left(-x \sin \theta_{i}^{\mathrm{B}}-z \cos \theta_{i}^{\mathrm{B}}\right)}\right\} e^{j \omega t} \\
-\dot{H}_{x i} & =\dot{H}_{x i}^{\mathrm{F}}+\dot{H}_{x i}^{\mathrm{B}} \\
& =\frac{\dot{\gamma}_{i}}{j \omega \dot{\mu}_{i}}\left\{\cos \theta_{i}^{\mathrm{F}} \dot{A}_{i} e^{-\dot{\gamma}_{i}\left(-x \sin \theta_{i}^{\mathrm{F}}+z \cos \theta_{i}^{\mathrm{F}}\right)}-\cos \theta_{i}^{\mathrm{B}} \dot{B}_{i} e^{-\dot{\gamma}_{i}\left(-x \sin \theta_{i}^{\mathrm{B}}-z \cos \theta_{i}^{\mathrm{B}}\right)}\right\} e^{j \omega t}
\end{aligned}
$$

On the other hand, the electromagnetic fields in medium 0 and medium $n+1$ are expressed as

$$
\begin{aligned}
& \dot{E}_{y 0}=\dot{E}_{y 0}^{\mathrm{I}}+\dot{E}_{y 0}^{\mathrm{R}}=\left\{\dot{A}_{0} e^{-\dot{\gamma}_{0}\left(-x \sin \theta_{0}^{\mathrm{I}}+z \cos \theta_{0}^{\mathrm{I}}\right)}+\dot{B}_{0} e^{-\dot{\gamma}_{0}\left(-x \sin \theta_{0}^{\mathrm{R}}-z \cos \theta_{0}^{\mathrm{R}}\right)}\right\} e^{j \omega t} \\
& -\dot{H}_{x 0}=\dot{H}_{x 0}^{\mathrm{I}}+\dot{H}_{x 0}^{\mathrm{R}} \\
& \quad=\frac{\dot{\gamma}_{0}}{j \omega \dot{\mu}_{0}}\left\{\cos \theta_{0}^{\mathrm{I}} \dot{A}_{0} e^{-\dot{\gamma}_{0}\left(-x \sin \theta_{0}^{\mathrm{I}}+z \cos \theta_{0}^{\mathrm{I}}\right)}-\cos \theta_{0}^{\mathrm{R}} \dot{B}_{0} e^{-\dot{\gamma}_{0}\left(-x \sin \theta_{0}^{\mathrm{R}}-z \cos \theta_{0}^{\mathrm{R}}\right)}\right\} e^{j \omega t} \\
& \dot{E}_{y n+1}=\dot{E}_{y n+1}^{\mathrm{T}}=\dot{A}_{n+1} e^{-\dot{\gamma}_{n+1}\left(-x \sin \theta_{n+1}^{\mathrm{T}}+z \cos \theta_{n+1}^{\mathrm{T}}\right)} e^{j \omega t} \\
& -\dot{H}_{x n+1}=\dot{H}_{x n+1}^{\mathrm{T}}=\frac{\dot{\gamma}_{n+1}}{j \omega \dot{\mu}_{n+1}} \cos \theta_{n+1}^{\mathrm{T}} \dot{A}_{n+1} e^{-\dot{\gamma}_{n+1}\left(x \sin \theta_{n+1}^{\mathrm{T}}+z \cos \theta_{n+1}^{\mathrm{T}}\right)} e^{j \omega t}
\end{aligned}
$$

where

$$
\dot{\gamma}_{i}=\sqrt{\left(j \omega \dot{\varepsilon}_{i}+\sigma_{i}\right)\left(j \omega \dot{\mu}_{i}\right)}, \quad(i=0 \sim n+1)
$$

In the above Eqs., $\dot{A}_{i}$ and $\dot{B}_{i}$ denote the amplitude factor, and $\dot{\gamma}_{i}$ denote the propagation factor.

\subsection{Reflection and transmission coefficients}

In this analysis, Snell's law is defined at each boundary because Eq. (4) is satisfied regardless of the $x$-coordinate. Then, the propagation angles of the electromagnetic fields in Eqs. (6) (11) are obtained as

$$
\begin{aligned}
& \theta_{0}^{\mathrm{I}}=\theta_{0}^{\mathrm{R}} \equiv \theta_{0} \quad, \quad \theta_{i}^{\mathrm{F}}=\theta_{i}^{\mathrm{B}} \equiv \theta_{i} \quad, \quad(i=1 \sim n) \\
& \theta_{i}=\cos ^{-1} \sqrt{1-\left(\frac{\dot{\gamma}_{0} \sin \theta_{0}}{\dot{\gamma}_{i}}\right)^{2}}, \quad 0 \leq \theta_{i} \leq \frac{\pi}{2}
\end{aligned}
$$

By substituting the electromagnetic field in each layer and medium into Eq. (4), the unknown coefficients $\dot{A}_{i}$ and $\dot{B}_{i}$ are expressed by using the following matrix representation: 


$$
\left.\begin{array}{l}
{\left[\begin{array}{c}
\dot{A}_{i} \\
\dot{B}_{i}
\end{array}\right]=\frac{1}{2} \dot{\mathbf{M}}_{i}^{\mathrm{TE}}\left[\begin{array}{c}
\dot{A}_{i+1} \\
\dot{B}_{i+1}
\end{array}\right], \quad(i=0 \sim n)} \\
\dot{B}_{n+1}=0
\end{array}\right\}
$$

where

$$
\left.\begin{array}{rl}
\dot{\mathbf{M}}_{i}^{\mathrm{TE}} & =\left[\begin{array}{ll}
\left(1+u_{i+1} / u_{i}\right) e^{\left(v_{i}-v_{i+1}\right) z_{i}} & \left(1-u_{i+1} / u_{i}\right) e^{\left(v_{i}+v_{i+1}\right) z_{i}} \\
\left(1-u_{i+1} / u_{i}\right) e^{-\left(v_{i}+v_{i+1}\right) z_{i}} & \left(1+u_{i+1} / u_{i}\right) e^{-\left(v_{i}-v_{i+1}\right) z_{i}}
\end{array}\right] \\
u_{i} & =\frac{\dot{\gamma}_{i}}{j \omega \dot{\mu}_{i}} \cos \theta_{i}, \quad v_{i}=\dot{\gamma}_{i} \cos \theta_{i}
\end{array}\right\}
$$

$\dot{\mathbf{M}}_{i}^{\mathrm{TE}}$ is the characteristic matrix that determines the propagation behavior of the electromagnetic noise in the TE mode at each boundary. Here, Eq. (14) is the recurrence equation for the amplitude factor of the electromagnetic noise in each layer and medium. In the recurrence equation, the product of the matrix $\dot{\mathbf{M}}_{i}^{\mathrm{TE}}$ is obtained as the following $2 \times 2$ square matrix:

$$
\prod_{i=0}^{n} \dot{\mathbf{M}}_{i}^{\mathrm{TE}}=\left[\begin{array}{ll}
\dot{m}_{11} & \dot{m}_{12} \\
\dot{m}_{21} & \dot{m}_{22}
\end{array}\right]
$$

Unknown coefficients $\dot{B}_{0}$ and $\dot{A}_{n+1}$ can be obtained from the recurrence equation when the amplitude factor $\dot{A}_{0}$ of the incident electromagnetic noise is provided. Thus, the reflection ratio $R_{\mathrm{r}}$ of the electromagnetic noise at $z=z_{0}$ and the transmission ratio $T_{\mathrm{r}}$ of the electromagnetic noise at $z=z_{n}$ can be derived as

$$
\begin{aligned}
& R_{\mathrm{r}}=\left.\left|\frac{E_{y 0}^{\mathrm{R}}}{E_{y 0}^{\mathrm{I}}}\right|\right|_{z=z_{0}}=\left|\frac{\dot{m}_{21} e^{2 \dot{\gamma}_{0} z_{0}}}{\dot{m}_{11}}\right| \\
& T_{\mathrm{r}}=\left|\frac{E_{y n+1}^{\mathrm{T}}}{E_{y 0}^{\mathrm{I}}}\right|_{z=z_{n}}=\left|\frac{2^{n+1} e^{-\left(\dot{y}_{n+1}-\dot{y}_{0}\right) z_{n}}}{\dot{m}_{11}}\right|
\end{aligned}
$$

Using Eqs. (17) and (18), the electromagnetic noise suppression effect of the FGM plate is defined as follows ${ }^{(3)}$ :

$$
S=1-\left(R_{\mathrm{r}}^{2}+T_{\mathrm{r}}^{2}\right)
$$

Moreover, Eqs. (17) and (18) are expressed by their decibel values and hence defined as the reflection coefficient $R_{\mathrm{dB}}$ and the transmission coefficient $T_{\mathrm{dB}}$ :

$$
\begin{aligned}
& R_{\mathrm{dB}}=-20 \log _{10}\left|R_{\mathrm{r}}\right| \\
& T_{\mathrm{dB}}=-20 \log _{10}\left|T_{\mathrm{r}}\right|
\end{aligned}
$$

\section{Results of the Numerical Calculation and Discussion}

\subsection{Conditions for the numerical calculation}

In order to determine the composition distribution in the FGM plate, a nondimensional thickness coordinate was introduced:

$$
\zeta=z / z_{n}
$$


The dielectric loss materials are designed as composite materials, which are composed of an insulator matrix (such as epoxy and polyester) and resistivity particles (such as titanium oxide and carbon black) ${ }^{(7),(8)}$. Hence, we will be able to select many materials with regard to the usage environment and from the viewpoint of practical applications. However, the empirical and theoretical formulas for obtaining the electromagnetic properties of these materials are very limited. In this numerical calculation, the matrix was assumed to be an epoxy resin and the resistivity particles were assumed to be titanium oxide particles because the electromagnetic properties of these materials have been clarified in the experiment ${ }^{(8)}$. From Ref. (7), the weight distribution $g$ (PHR ; Parts per Hundred parts of Resin) of resistivity particles through the plate thickness is defined as a power function of $\zeta$ :

$$
g(\zeta)=g_{\max } \cdot \zeta^{\eta}
$$

Here, $g_{\max }$ denotes the PHR of resistivity particles at $z=z_{n}$ and $\eta$ is the distribution parameter. The case of $\eta=1$ represents linear distribution. The distributions of the resistivity particles used in this numerical calculation are shown in Fig. 2. The complex relative permittivity in the FGM plate is calculated by using the following equation on the basis of the empirical formula ${ }^{(8)}$ :

$$
\dot{\varepsilon}_{\mathrm{r}}(g)=3.50+0.40 g^{1.05}-j\left(0.10+0.15 g^{1.15}\right), \quad 0 \leq g \leq 30
$$

Here, we define $g_{\max }$ to be 30 PHR because the contents of titanium oxide in the referred experiment have $g_{\max }$ values in the range of up to 32 PHR. The relationship between the complex relative permittivity and PHR of the particles is shown in Fig. 3.

In order to examine the relationship between the number of layers and the solution accuracy of this approximation analysis, the solution for the FGM plate that is divided into 1000 layers is defined as the standard solution. For all the distributions in this numerical

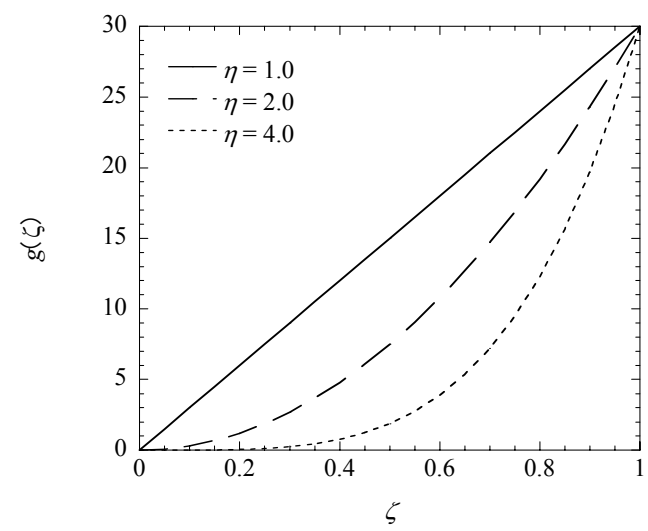

Fig. 2 Composition distribution of resistivity particles

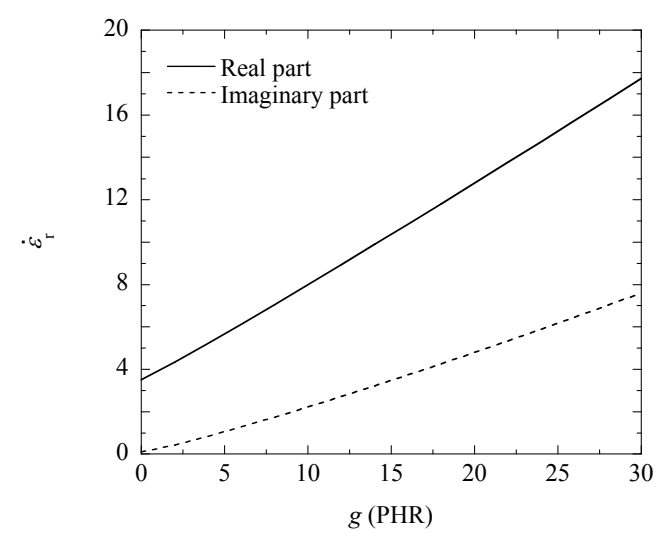

Fig. 3 Relationship between complex relative permittivity and PHR of resistivity particles 
calculation, the relative error between the solution with 20 layers and the standard solution is $\leqq 1 \%$. Therefore, the FGM plates are approximated as multilayered plates that are divided into 20 layers with distinct homogeneous properties in each layer. With regard to the electromagnetic noise defined in this paper, a large number of faulty operation and radar false echoes in the radar frequency band have been reported. The radar frequency band is $2 \mathrm{GHz} \sim 18 \mathrm{GHz}$. In this numerical calculation, the center frequency of the radar frequency band is $10 \mathrm{GHz}$. Further, medium 0 and medium $n+1$ are in free space.

\subsection{Design objective of electromagnetic noise suppression}

In the reports on the properties of electromagnetic wave absorption of electromagnetic noise reflected from a metal structure, the design objective is to reduce the reflected noise by $90 \%{ }^{(3),(6)}$. On the other hand, in the reports on the properties of electromagnetic wave shield for transmitted waves, the design objective is to reduce the transmitted noise by $90 \%{ }^{(9),(10)}$. Generally, the suppression effect of reflected noise is not often discussed during the evaluation of the properties of electromagnetic wave shield; when discussed, this suppression effect is $\leqq 20 \%{ }^{(10)}$. It is very difficult to suppress both the reflected noise and the transmitted noise by $\geqq 90 \%$ without the occurrence of total reflection by the metal structure; moreover, such occurrences have not been reported so far. One of the design objectives in this study is the performance of the suppression of either reflected noise or transmitted noise by $\geqq 90 \%$ and the suppression of the other noise by $\geqq 50 \%$ for electromagnetic noise of a specific frequency.

\subsection{Quantitative evaluation of electromagnetic noise suppression}

Figure 4 shows the effect of electromagnetic noise suppression, the reflection coefficient and transmission coefficient of the FGM plate with the distribution parameter $\eta=1.0$, 2.0, 4.0 and plate thickness $0.1 \mathrm{~mm} \sim 10.0 \mathrm{~mm}$. In the results shown here, the incident electromagnetic noise is perpendicular to the plate. For comparing the electromagnetic noise suppression in the FGM plate with that in the homogeneous plate, the PHR of resistivity particles in the homogeneous plate is represented by $g_{0}$, and the numerical calculation results for the homogeneous plate with $g_{0}=10,15,20$ PHR are shown in Fig. 5. By comparing Fig. 4(a) with Fig. 5(a), the electromagnetic noise suppression effect in the FGM plate with plate thickness in the range $4.5 \mathrm{~mm} \sim 10.0 \mathrm{~mm}$ is improved by approximately $15 \% \sim 25 \%$ as compared to that of the homogeneous plate. The dominant mechanism in the electromagnetic noise suppression is the attenuation of noises due to the energy conversion inside the plate, the initial reflection of noises due to the impedance mismatch at the boundary of the plate and free space, and the multiple interference of noises inside the plate. This mechanism is realized by controlling the phase and attenuation of the electromagnetic noises by the adequate selection of the thickness and material composition of the plate.

Figures 4(b), 4(c) and Figs. 5(b), 5(c) show the reflection coefficient and the transmission coefficient of the FGM plate and the homogeneous plate, respectively. High $\mathrm{dB}$ values is more effective in the suppression of the noise. A value of approximately $6 \mathrm{~dB}$ represents the suppression of the noise by $50 \%$, and $20 \mathrm{~dB}$ represents the suppression of the noise by $90 \%$. In all the Figs., the extreme value is obtained for a certain plate thickness because the effect of plate thickness on the multiple interference is remarkable. In the FGM plate shown in Fig. 4(b), the reflection coefficient for plate thickness in the range $5.0 \mathrm{~mm} \sim$ $6.0 \mathrm{~mm}$ is remarkably higher than that of the homogeneous plate because the noises are suppressed by the initial reflection and the multiple interference. The surface $\left(z=z_{0}\right)$ of all the FGM plates in this numerical calculation has the same material composition. Therefore, the reflection coefficient of the FGM plate with $\eta=2.0$ is high, particularly because the material composition distribution has a large effect on the multiple interference. 


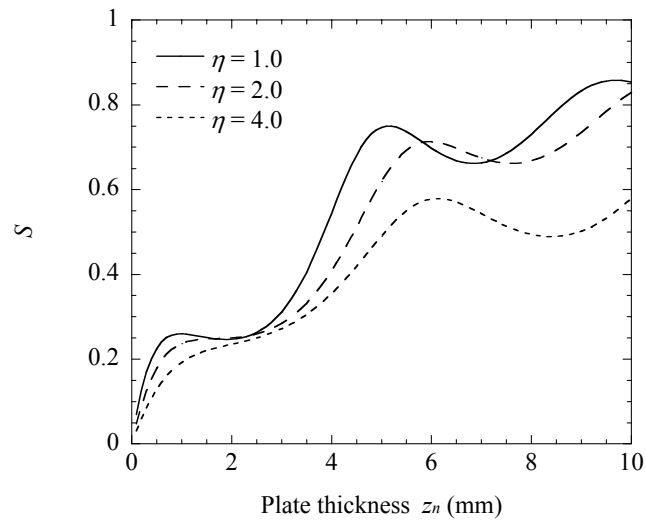

(a) Electromagnetic noise suppression effect

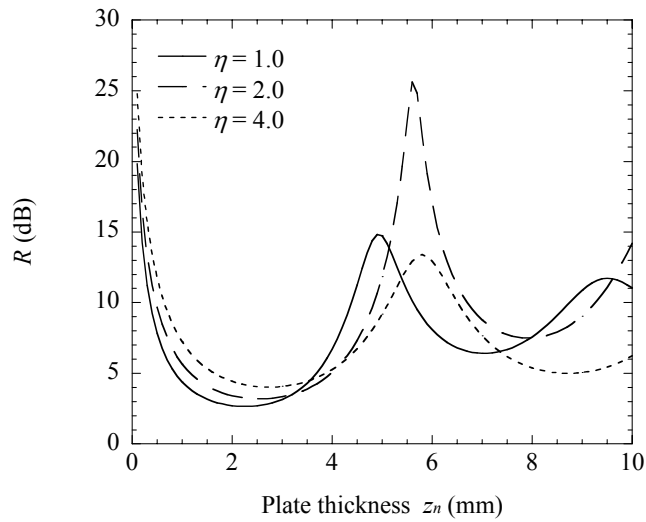

(b) Reflection coefficient

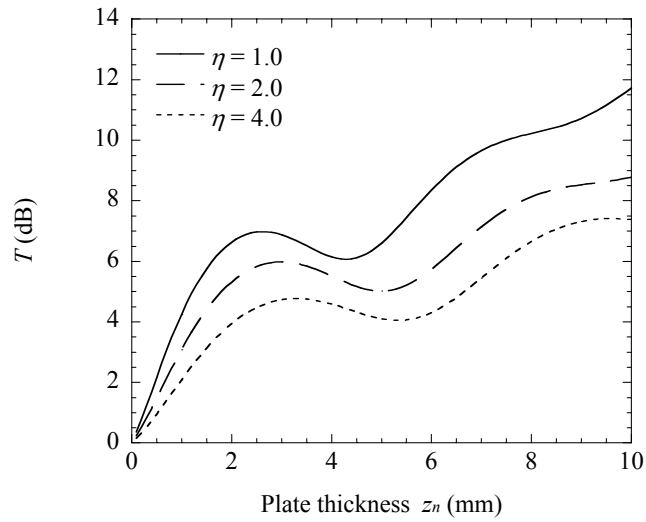

(c) Transmission coefficient

Fig. 4 Electromagnetic noise suppression in the FGM plates $\left(\theta_{0}^{\mathrm{I}}=0^{\circ}\right)$

Comparing Fig. 4(c) with Fig. 5(c), it is observed that the difference between the two transmission coefficients is small. In the range of the frequency band and plate thickness used for the numerical calculations in this study, the graded composition distribution and the plate thickness particularly have significant effect on the reflection ratio. The reason for the above tendency can be explained by using Eqs. (17) and (18). In the transmission ratio in Eq. (18), the element of the matrix that governs the suppression of electromagnetic noise exists only in the denominator. Hence, the decrease in transmission ratio is basically related to the increase in plate thickness and PHR of resistivity particles in the FGM plate. On the other hand, in the reflection ratio in Eq. (17), the element of the matrix that governs the suppression of electromagnetic noise exists in both the denominator and the numerator. Hence, the plate thickness and composition distribution configuration of the FGM plate have a complex effect on the decrease in the reflection ratio. 


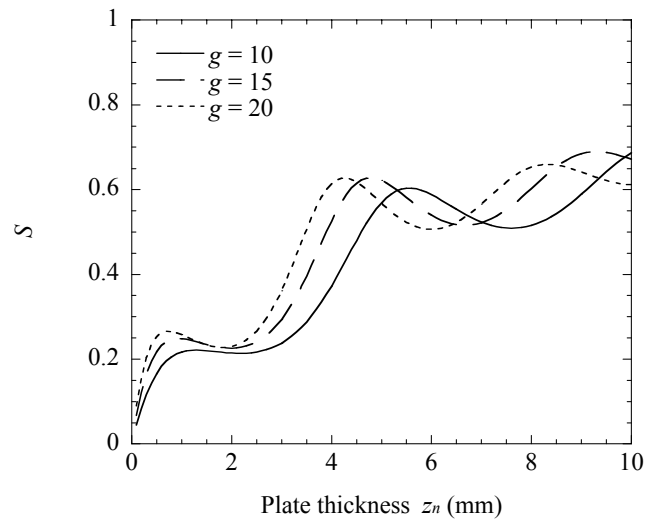

(a) Electromagnetic noise suppression effect

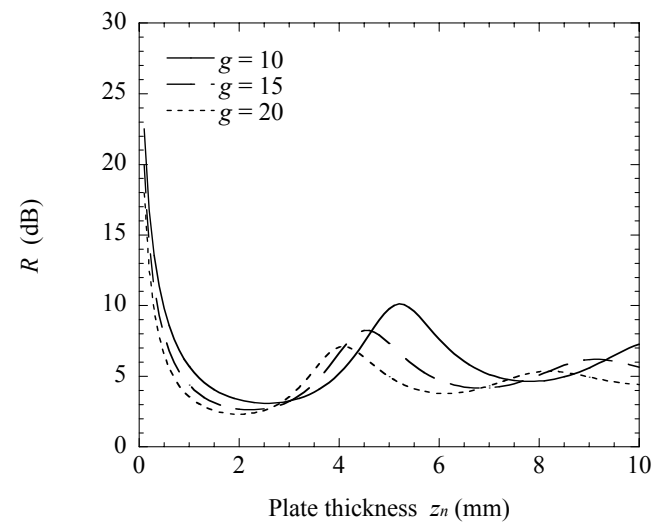

(b) Reflection coefficient

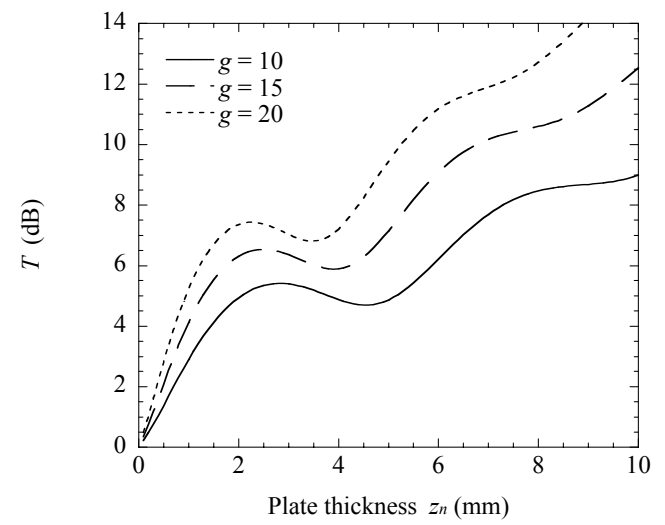

(c) Transmission coefficient

Fig. 5 Electromagnetic noise suppression in the homogeneous plates $\left(\theta_{0}^{\mathrm{I}}=0^{\circ}\right)$

We consider the material design for the suppression of both the reflected noise and the transmitted noise. For the suppression of reflected noise, the material design that suppresses the reflected noise by $\geqq 90 \%$ and the transmitted noise by approximately $45 \%$ can be realized in the case of the FGM plate with distribution parameter $\eta=2.0$ and plate thickness $5.4 \mathrm{~mm} \sim 5.9 \mathrm{~mm}$. This result is close to the performance objective of the design model, and it is concluded that we have achieved a satisfactory result for the suppression of electromagnetic noise.

In recent years, measures for the suppression of noise in high-frequency bands that are higher than the radar frequency band have become necessary because of the rapid high-speed digitalization of electric devices. In this case, because of the short wavelength of the noise, the effect of multiple interference will be exerted by thinner plates as compared with the results of this study. Therefore, it is inferred that the electromagnetic noise 


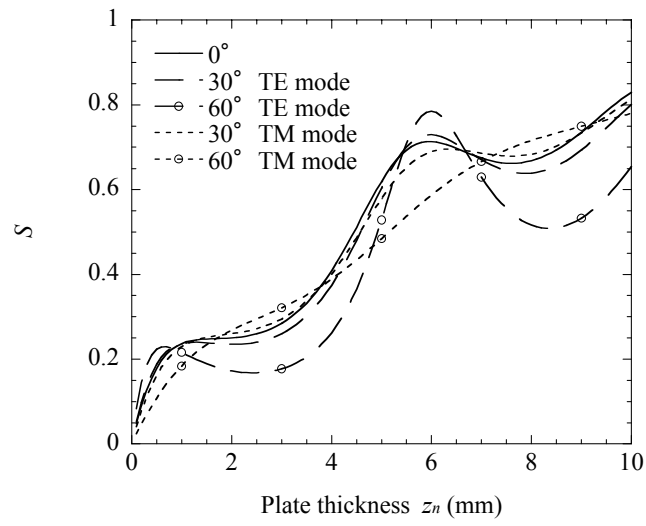

(a) Electromagnetic noise suppression effect

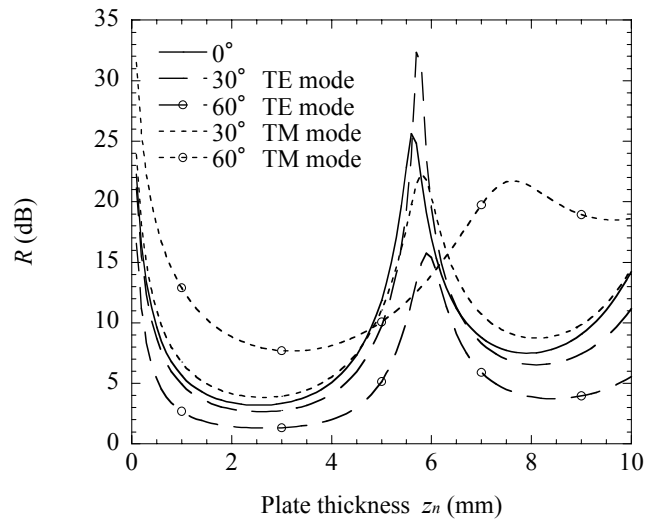

(b) Reflection coefficient

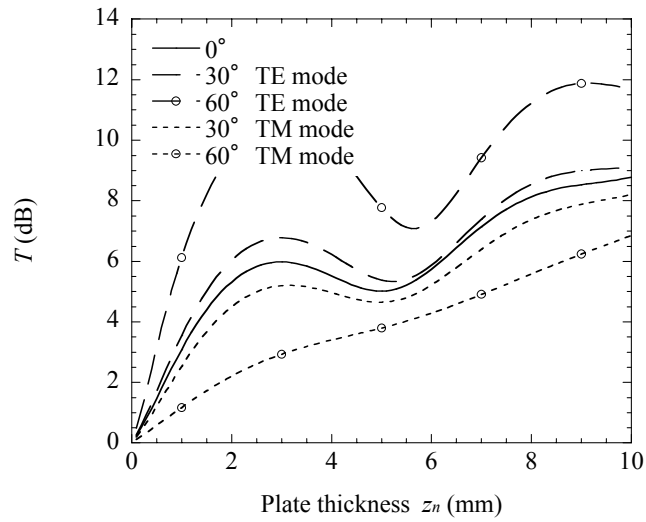

(c) Transmission coefficient

Fig. 6 Polarization characteristic of electromagnetic noise suppression in the FGM plate $(\eta=2.0)$

suppression effect achieved due to the thinner plate thickness.

Figure 6 shows the polarization characteristics of the electromagnetic noise suppression in the FGM plate with the distribution parameter $\eta=2.0$. Here, the incident angles of the electromagnetic noise are $\theta_{0}^{\mathrm{I}}=0^{\circ}, 30^{\circ}$, and $60^{\circ}$. The results for the incident angle $30^{\circ}$, except the peak value in the reflection coefficient in the TE mode, vary by approximately $10 \%$ in comparison to the results for the vertical incidence. On the other hand, the results for the incident angle $60^{\circ}$ vary by up to $50 \%$ in comparison to the results for the vertical incidence. From these results, it is understood that the effect of electromagnetic noise suppression on the plate with a large curvature is significantly different from that on a flat plate, which is used in this study.

From the experimental results obtained by Liu et al. ${ }^{(7)}$ and the present numerical calculation results, it can be inferred that adequate increase in the PHR of the resistivity 
particles yields thinner plate thickness and improves the effect of electromagnetic noise suppression. The FGM plate with $g_{\max }=60$ PHR was considered to be one of the examination cases. As a result, the plate thickness that showed the peak value of the reflection coefficient became thin by approximately $1 \mathrm{~mm}$ as compared to that of the FGM plate with $g_{\max }=30 \mathrm{PHR}$, and further the value of the transmission coefficient increased. With regard to the tendency of the electromagnetic noise suppression effect, the graded composition distribution that increases the PHR of resistivity particles on the $z=z_{n}$ side is effective.

Thus far, the authors have reported on the analysis of hygrothermoelastic problems in resin materials ${ }^{(11)}$ and the analysis of hygrothermal stresses in FGM plates ${ }^{(12)}$. From these reports and the results in this paper, we regard that the material design suitable for both the suppression of electromagnetic noise and the reduction in hygrothermal stress will be possible by the appropriate constituent materials and graded composition distribution.

\section{Conclusions}

In this paper, we have presented a method for the material composition design of FGM plates with the function of electromagnetic noise suppression. For analyzing the electromagnetic field in the FGM plate with arbitrary nonhomogeneity of composition distribution, the FGM plate was considered to be a multilayered plate in which the electromagnetic properties are homogeneous inside each layer. The analytical solutions for electromagnetic noise suppression by using the solution to the electromagnetic fields in the multilayered plate were derived. The numerical calculations were carried out for epoxy resin/titanium oxide FGM plates with a graded composition distribution expressed in the form of a power function. As a result, the FGM plate with the function of electromagnetic noise suppression close to the design objective was designed by controlling the graded composition distribution and plate thickness.

If the electromagnetic properties can be obtained by using the experimental formula, the power law of Lichtenecker ${ }^{(13)}$, etc., the material design for the electromagnetic noise suppression in the FGM plates composed of other constituent materials can easily be achieved by the present method. In further studies in the future, we consider that the material design of FGMs with regard to the temperature dependence and humidity dependence of electromagnetic properties is necessary for applications under heat and humidity environments.

\section{Acknowledgment}

The authors would like to thank Prof. M. Kominami, Osaka Electro-Communication University, for providing valuable data with respect to the electromagnetic properties.

\section{References}

(1) Hondou, T., A Remake on the Public Exposure to Mobile Phones (Review), Butsuri (in Japanese), Vol.58, No.6 (2003), pp.430-434.

(2) Tsugawa, S., Communication Systems in the Intelligent Transport Systems, The transactions of the Institute of Electronics, Information and Communication Engineers, $B$, Vol.J82-B, No.11 (1999), pp.1958-1965.

(3) Sugimoto, S., Recent Trends in the Development of Microwave Absorption Materials (Review), Journal of the Magnetics Society of Japan, Vol.27, No.8 (2003), pp.862-869.

(4) Shimizu, Y. et al. Denjiha no Kyusyu to Syahei (in Japanese), (1989), p.88, 222, 443, Nikkei Gijyutu Tosyo, Tokyo. 
(5) M. S. Pinho, et al., Aging Effect on the Reflectivity Measurements of Polychloroprene Matrices Containing Carbon Black and Carbonyl-iron Powder, Polymer degradation and stability, Vol.43, No.1 (2001), pp.1-6.

(6) Kato, M., et al., Analytical Study on Variation of Temperature and Absorption Characteristics of Single-Layer Radiowave Absorber by Irradiation Electric Power, Microwave and Optical Technology Letters, Vol.41, No.1 (2004), pp.3-5.

(7) H. T. Liu., et al., Design of Functionally Graded Materials towards RAM and Their Microwave Reflectivity, Materials Science Forum, Vol.423- 425 (2003), pp.427-430.

(8) Soh, T., et al., A Study on Relationship between Applied Frequency and Thickness of Single Layer Absorber Using Epoxy Resin Including Titanium Oxide and Carbon Particles, The Journal of the Institute of Electronics, Information and Communication Engineers, B, Vol.J84-B, No.10 (2001), pp.1901-1904.

(9) Miura, K. and Miyazaki, Y., Electromagnetic Shielding Properties of Lossy Composite Multi layers, The Institute of Electronics, Information and Communication Engineers technical report. Electromagnetic compatibility, EMCJ91-66 (1991), pp.7-14.

(10) Murakami, R., et al., Fundamental Characteristics of Shielding Effectiveness for Electromagnetic Wave of Plastic Composite Oriented Fine Copper Wire, Transactions of the Japan Society of Mechanical Engineers, Series A, Vol.66, No.642 (2000), pp.291-297.

(11) Sugano, Y. and Chuuman, Y., Analytical Solution of Transient Hygrothermoelastic Problem due to Coupled Heat and Moisture Diffusion in a Hollow Circular Cylinder, Transactions of the Japan Society of Mechanical Engineers, Series A, Vol.59, No.564 (1993), pp.1956-1963.

(12) Chiba, R. and Sugano, Y., Hygrothermal Stress Analysis and Material Composition Optimization of Functionally Graded Plates, Proceedings of the Computational Mechanics Conference of the Japan Society of Mechanical Engineers, No.15 (2002-10), pp.455-456.

(13) Ogasawara, N., Denki Zairyo (in Japanese), (1979), p.22, Rikogakusha Publishing Co., Ltd. 